Journal of Science and Science Education

http://jppipa.unram.ac.id/index.php/jossed/index

\title{
Assessment of Infertility Factors and Their Relative Impact in Pregnancy using Ultrasonography \& Hormonal Checkup
}

\author{
Mohamed Elfadil M. Gar-elnabi1,2, Manal Z. Abdelsalam¹, Mohamed A. Eltahir ${ }^{3}$, \\ Mohammed A. Ali Omer $14^{*}$ and Mustafa J. Musa ${ }^{5}$
}

${ }^{1}$ College of Medical Radiologic Science, Sudan University of Science \& Technology, Khartoum-Sudan

${ }^{2}$ Faculty of Radiography \& Medical Imaging Science, National University, Khartoum- Sudan

${ }^{3}$ Al-Ghad International Colleges for Applied Medical Sciences, Qassim, KSA

${ }^{4}$ Department of Radiologic Technology, College of Applied Medical Science, Qassim University, KSA

${ }^{5}$ Department of Radiologic Technology, College of Applied Medical Science, University of Jeddah, KSA

\section{Article Info}

Received: August 21th, 2020

Revised: September 30th, 2020

Accepted: October $8^{\text {th }}, 2020$

\begin{abstract}
Infertility as a social and psychological problem among middle east natives. Among them, Transvaginal ultrasound (TVU) and hormonal tests have been selected to evaluate infertility in this study. The objective was to assess the infertility factors and their relative impact in pregnancy and to deduce the equation to predict infertility. Methods: a retrospective data (morphometric pathologies of uterus and ovaries using TVU and laboratory hormones (FSH, LH)) have been collected from clinics for 180 women of reproductive age (15-49 years) in Sudan. The data analyzed by SPSS. The results showed that there were $120(66.7 \%)$ infertile and 60 (33.3\%) fertile ladies based on the marriage date. The common ovarian causes of infertility were the polycystic ovary (PCOs) $23.3 \%$, simple cyst $6.1 \%$, hemorrhagic cyst, $4.4 \%$, and uterus causes: $6.7 \%$ intramural fibroma, $6.1 \%$ retroverted uterus, $2.8 \%$ submural fibroma, 2.2 polyps. The general accuracy of Stepwise Linear Discriminant Analysis (SLDA) was 78.9\%, for infertile was 70.8\% and for fertile 95\%. Larger ovarian width indicates significance $(p<0.05)$ infertility and FSH level low among infertile ladies, but LH is less dependent on discrimination.
\end{abstract}

Keywords: Fertility, Infertility, Morphometric, Ultrasonography

Citation: Gar-elnabi, M.E.M., Abdelsalam, M.Z., Eltahir, M.A., Omer, M.A.A., \& Musa, M.J. (2020). Assessment of Infertility Factors and Their Relative Impact in Pregnancy using Ultrasonography \& Hormonal Checkup. Journal of Science and Science Education (JoSSEd), 1(1): 30-34.

\section{Introduction}

Infertility denotes a failure to conceive within one or more years of regular unprotected coitus [1]. It has been one of most social and psychological problems such as stress and depression [2] for both genders, especially among Muslims and or middle east natives; and more over it limits the existence of mankind over the earth. Female Infertility factors represent $20 \%$ to $70 \%$, and males represent $2.5 \%$ to $12 \%$. Infertility has been recorded at the highest rate in Africa and Central/Eastern Europe. And based on a variety of sources, rates of male infertility in North America,
Australia, and Central and Eastern Europe varied from $4.5-6 \%, 9 \%$, and $8-12 \%$, respectively [3]. The common induction factors for infertility have been highlighted by many scholars; [4, 9], that classified to (i) Ovarian factors; that include Polycystic Ovaries (PCO) and (ii) the common risk factors that include: reproductive (infertility, pregnancy-related risks), metabolic (obesity, insulin resistance (IR), gestational and type II diabetes (DM2) and cardiovascular risk factors) and (iii) psychological features (anxiety and depression, impaired quality of life, body image and eating disorders. In addition to (iv) chronic pelvic inflammatory disease, fibroids, anatomical problems, 
endometrial/cervical polyp, free fluid in pelvic/abdominal cavity, endometritis, endometriosis, adenomyosis, chocolate cyst, tubo-ovarian mass, intrauterine and intrapelvic adhesions, septate uterus as well as pelvic abscess [4].

The hormonal indexes related to infertilities have been highlighted by Williams [5], as the value of serum progesterone of $>30 \mathrm{nmol} /$ liter is considered proof of adequate ovulation, although the WHO uses 18 $\mathrm{nmol} /$ liter to confirm ovulation. Such test done on day 2-4 of the cycle (if there is one), which identify four essential causes of ovulatory failure, such as:

Normogonadotrophic anovulation,

Hyperprolactinemia,

Hypogonadotropic hypogonadism, and

Hypergonadotropic hypogonadism.

Regarding Follicular Stimulating Hormone (FSH); values are checked on day 2 to day 4 with a cutoff point of $12 \mathrm{IU} /$ liter and should be not $<5$ $\mathrm{mIU} / \mathrm{ml}$; as the lower limit could be induced by hypogonadotrophic hypogonadism, which is usually a result of primary hypothalamic or pituitary failure in addition to excessive stress/exercise, malnutrition, or low weight. Hypergonadotrophic hypogonadism is defined by raised FSH concentrations $(>20 \mathrm{mIU} / \mathrm{ml})$ and indicates ovarian failure. The increment of FSH above $>50 \mathrm{IU} / \mathrm{ml}$ is considered as a diagnostic index for ovarian failure; however, most patients with oligomenorrhoea or amenorrhoea will have normal gonadotrophins as part of the polycystic ovarian syndrome (PCOS).

The normal Prolactin hormone $(\mathrm{PH})$ range is 600- $800 \mathrm{mIU} / \mathrm{ml}$, as estrogen is normal [6]. Hence the increment of $\mathrm{PH}$ could be ascribed to pregnancy, pituitary prolactinoma, stalk compression by hypothalamic or pituitary tumors, thyroid failure, PCOS, psychotherapeutic medication, or other pathologies as mentioned by Illions [7]. To reveal and diagnose infertility among female; there have been several methods, including normal menstrual cycle, sufficient normal level of hormones, ovulation, hysterosalpingography (less common), A laparoscopy, A pelvic ultrasound, and genetic testing may be necessary to detect a genetic origin for infertility [8].

Ultrasound is accepted as an essential imaging modality for evaluating and monitoring the reproductive system for diagnosis and screening purposes and follow-up of the normal stimulated cycle.
More than any other new method, ultrasound has made significant improvements in female infertility's modern management, especially in predicting ovulation and detection of certain ovulatory disorders [9].

Sonography has a vital role in depicting follicular development in patients treated for infertility and receiving ovulation induction medication. Although the follicle size can indirectly infer the maturity of the oocytes, the sonographic information can be couple with estradiol values to provide an accurate assessment of the presence or absence and a number of mature follicular. The anatomic information obtained with sonography concerning the size and development of maturing follicular can be used to distinguish physiologic from insufficient or abnormal cycles [10]. Relative to all mentioned above methods of investigations, endovaginally ultrasonography has been utilized more commonly recently due to numerous advantages such as: noninvasive, accuracy $84.1 \%$, sensitivity $68.2 \%$, specificity $91.5 \%$, positive predictive value $79 \%$, and $86 \%$ negative predictive value [11].

Authors have considered the current study among Sudanese ladies for the common cases encountered in clinical centers. The essential aim is to assess the infertility factors and their relative impact in pregnancy depending on endovaginally ultrasonography hormonal tests.

\section{Method}

This study was carried out as cross-sectional, descriptive, and direct analytical prospective data (Age, height, weight, echogenicity, texture, size, and level of female hormones) using an ultrasound system (Toshiba Xario XG SSA-680A) and laboratory hormonal test. The sample size was 180 patients referred to ultrasound clinics in Sudan from December 2017 to December 2018. For a transvaginal ultrasound exam, the women lie down on a table with her knees bent her feet may be held in stirrups. The probe was covered with a sterile condom, and a gel was then induced gently inside the vagina. The health care provider moves and kicking the probe around the area to prospect the relative anatomical and pathological information in the uterus, fallopian tubes, and ovaries. And with the enhancement of ultrasound caliber, the morphometric 
parameters of visualized pathologies, womb and ovaries have been assessed (Figures 1).

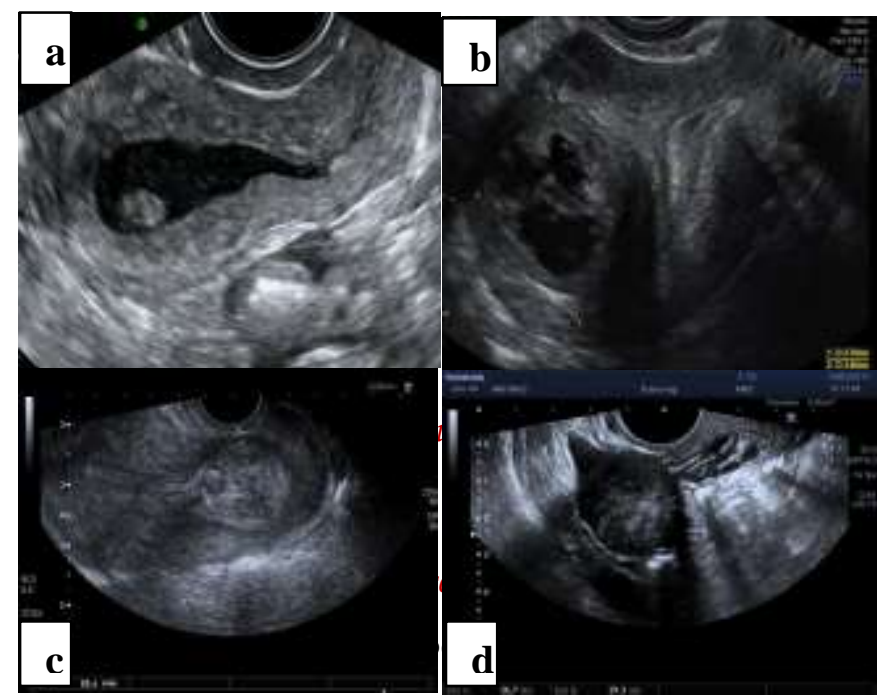

Figure 1: (a) Endometrial polyp, (b) Fibroid with cystic changes measured $3.7 \times 3.6 \mathrm{~cm}$, (c) Endometrial leiomyomata $(28.6 \mathrm{~mm})$ attached to the myometrium by a pedicle and (d) Endometrium fibroids with cystic changes measured $3.7 \times 3.6$ $\mathrm{cm}$.

The hormonal test results have been obtained as retrospective data from the patients' files. And the data analyzed using Excel software and linear discriminant analysis stepwise method, then the results presented in the form of bars, tables in addition to t-test.

\section{Result and Discussion}

Figure: 2, shows the distribution of ovarian pathologies finding using TVU. From the total sample of 180 females, $120(66.7 \%)$ ladies were considered infertile based on the marriage date. The ultrasound scan revealed that: out of 120 infertile ladies; there was $30.6 \%$ have normal ovaries, $23.3 \%$ have Polycystic Ovarian Syndrome PCOs, 6.1\% have Simple Cyst, 4.4\% have Hemorrhagic Cyst, 1.1\% have Endometrioma, and $1.1 \%$ have Dermoid Cyst. While ultrasound scanning for the fertile rest of sample $60(33.3 \%)$ ladies revealed that: the normal ladies represent $32.2 \%$, and simple cyst $1.1 \%$. These findings represent the same viable infertility factors nominated by Hussain \& Das [12]. PCOs have a high percentage (23.3\%) among infertile ladies, as it could cause an increased amount of testosterone and LH and decrease uptake of glucose by muscle, fat, and liver cells, resulting in large amounts of insulin by the pancreas. Low follicular stimulating hormones (FSH) levels also hinder the production of eggs from the ovarian follicles and form fluid-filled ovarian cysts that eventually cover the whole ovaries and prevent conception [13].

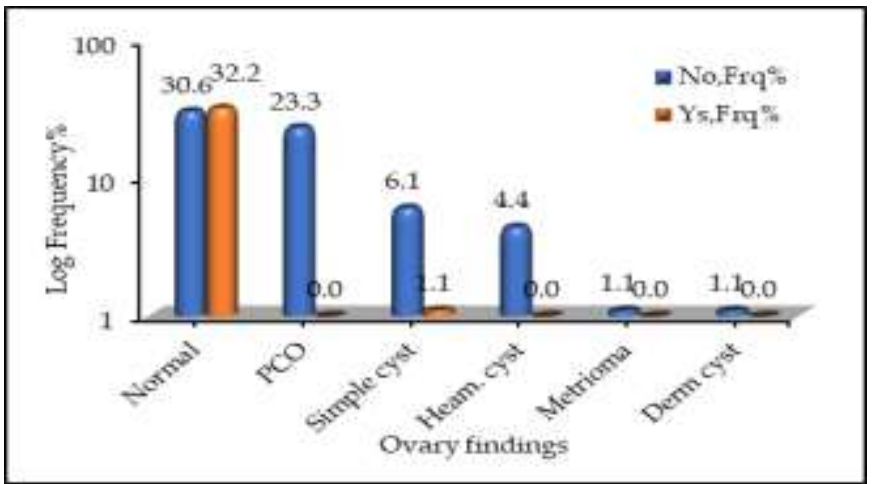

Figure: 2 Shows the percentage distribution of ovarian ultrasound findings in respect to fertility status

Parallel to these findings, ultrasound scanning of the uterus (Figure: 3 ) showed that: among the infertile ladies $120(66.7 \%)$, there were $44.4 \%$ as normal uterus, $1.7 \%$ as subserous fibroma, $7.6 \%$ intramural fibroma, $2.8 \%$ sub-mural fibroma, $2.2 \%$ polyps, $0.6 \%$ bicornuate uterus, $1.1 \%$ adenomyosis and $6.1 \%$ retroverted uterus. While ultrasound scanning for the fertile rest of sample $60(33.3 \%)$ ladies revealed that: the normal ladies represent $31.1 \%$, and subserous fibroma $0.6 \%$ and $1.7 \%$ have intramural fibroma. Relative to these findings; intramural fibroma $(7.6 \%)$ and retroverted uterus (6.1\%) attributed with the high incidence of infertilities as they cause distortion of the uterine cavity resulted in the decreased rates of clinical pregnancy, implantation, and ongoing pregnancy/live birth, as well as an increased rate of spontaneous miscarriage [14].



Figure: 3, Shows the percentage distribution of ultrasound uterus findings in respect to fertility status

To classify the female as fertile and infertile using SLDA method, the program chose four variables 
as the most discriminant ones: FSH, LH, Rt ovary width, and Lt ovary length (Table: 1). The overall classification accuracy was $78.9 \%$, for infertile was $70.8 \%$ and $95 \%$ for the fertile one. The width value of the Rt ovary separates the fertile from the infertile significantly $(p<0.05)$ in average; where the ovarian width for the infertile were bigger than the fertile one (Figure 4-a), as well as the Lt ovary length, which shows that the ovary length of the infertile female were bigger than that of the fertile one (Figure 4-b). In case of hormones; FSH also discriminates between the fertile and infertile; where the values of this hormones were lower among infertile female compared with fertile one (Figure 5-a), while LH (Fig. 5-b) showed wide variability for the fertile ones as well as low value in average than the infertile one. Still, in coordination with the other variable, it helps in the discrimination between the two status. Based on integrating the results of both ultrasound and laboratory exams using SLDA, the differentiation/classification between fertile and infertile female could be successfully derived by the following quantified equations:

Fertile $=(0.19 \times$ FSH $)+(-0.042 \times$ LH $)+(0.597 \times$ Rt anary width $)+(0.378 \times$ Ltenary length $)-15.07$

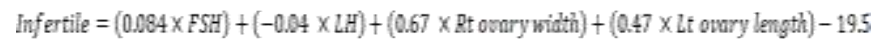

The vote will be to the higher values; i.e. the four values substituted in the two equations simultaneously and the bigger output defines the despondence's fertility.

Table 1. Shows the Stepwise Linear Discriminant Analysis (SLDA) to classify women as fertile or infertile using two ultrasound parameters and two laboratory tests.

\begin{tabular}{lll}
\hline \multirow{2}{*}{ Variables } & Fertility & \\
\cline { 2 - 3 } & Yes & No \\
\hline FSH & 0.190 & 0.084 \\
LH & -0.042 & 0.040 \\
Rt ovary width & 0.597 & 0.669 \\
Lt ovary Length & 0.378 & 0.465 \\
(Constant) & -15.072 & -19.499 \\
Fisher's linear discriminant functions & \\
\hline
\end{tabular}

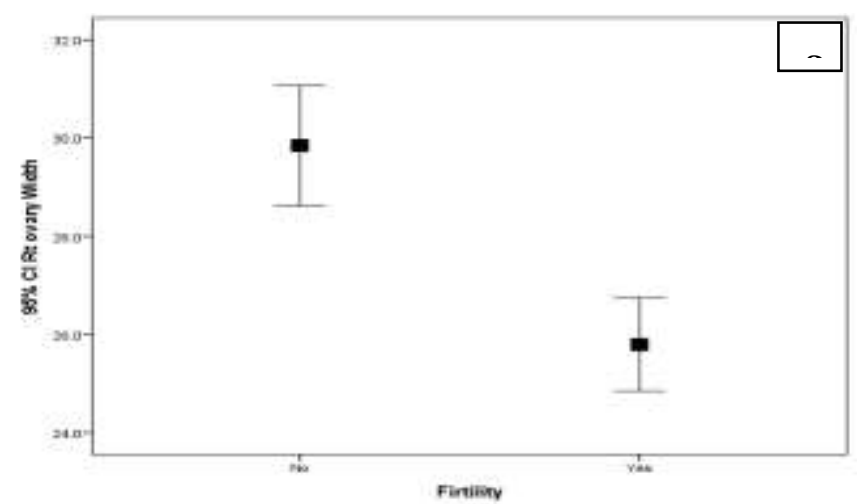

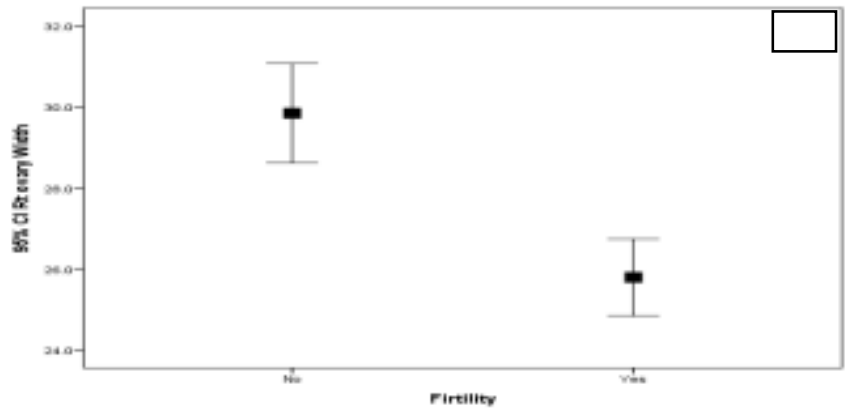
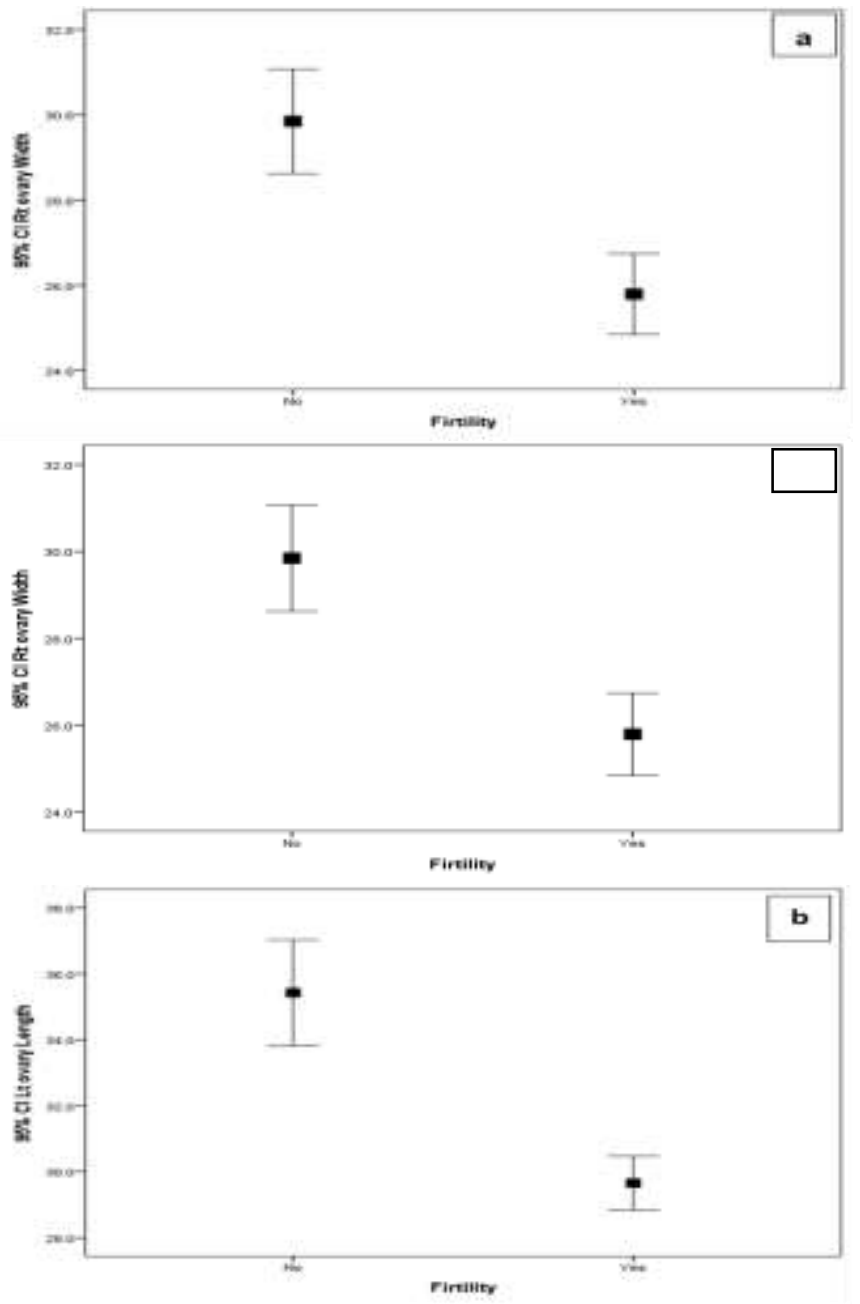

Figure 4: Error bar plot shows the discriminant power of (a) Rt ovary width and (b) Lt ovary length in discrimination between the fertile and non-fertile women using ultrasound caliber.

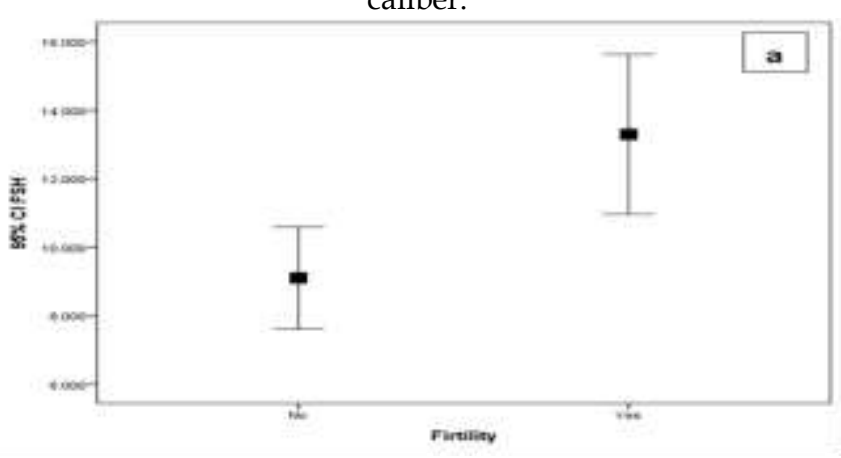




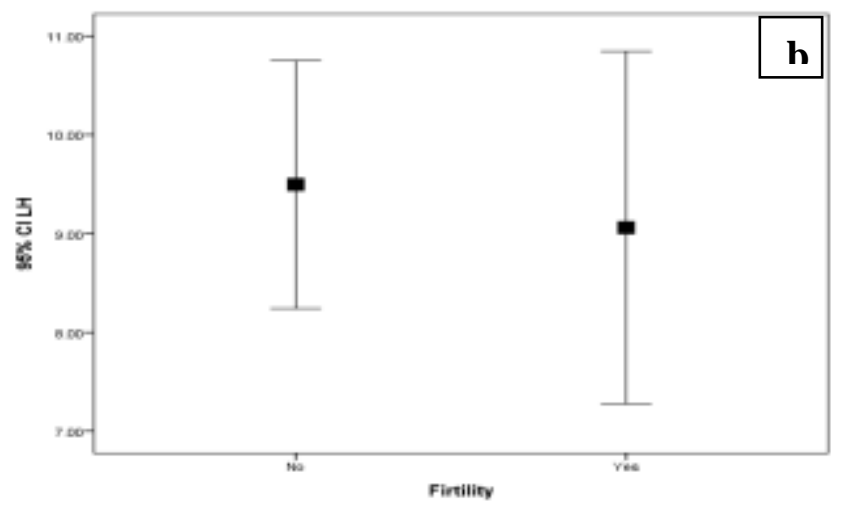

Figure 5: Error bar plot show the discriminant power of (a) FSH and (b) LH discrimination between the fertile and nonfertile women using laboratory test in coordination with ultrasound caliber

\section{Conclusion}

The differentiation between fertile and infertile females is still a challenging issue, but this study makes it possible to predict the infertile ladies based on the ovarian morphology and quantify the fertility/infertility in percent depending on an equation derived from the SLDA method.

\section{References}

[1] Kamel R. M. (2010). Management of the infertile couple: an evidence-based protocol. Reproductive biology and endocrinology: RBEE, 8, 21. https://doi.org/10.1186/1477-7827-8-21

[2] Fisher, J. R., \& Hammarberg, K. (2012). Psychological and social aspects of infertility in men: an overview of the evidence and implications for psychologically informed clinical care and future research. Asian journal of andrology, 14(1), 121-129. https://doi.org/10.1038/aja.2011.72

[3] Agarwal, A., Mulgund, A., Hamada, A., \&Chyatte, M. R. 2015. A unique view on male infertility around the globe. Reproductive biology and endocrinology, 13(1), 37. https://doi.org/10.1186/s12958-015-0032-1

[4] Teede, H., Misso, M., Deeks, A., Moran, L., Stuckey, B., Wong, J., ... Costello, M. (2011). Assessment and management of polycystic ovary syndrome: summary of an evidence-based guideline. Medical Journal of Australia, 195(S6), 65-113. https://doi.org/10.5694/mja2.2011.195.issue-S6

[5] Williams, C., Giannopoulos, T., \& Sherriff, E. A. (2003). Investigation of infertility with the emphasis on laboratory testing and with reference to radiological imaging. Journal of Clinical Pathology,
56(4),

$261-267$.

https://doi.org/10.1136/jcp.56.4.261

[6] Speroff L, Glass H, Kase G. 1994. Clinical gynecologic endocrinology and infertility, 5th. ed. Baltimore: Williams \& Wilkins.

[7] Illions, E. H., Valley, M. T., \& Kaunitz, A. M. 1998. Infertility. A clinical guide for the internist. The Medical clinics of North America, 82(2), 271-295. https://doi.org/10.1016/s0025-7125(05)70607-6

[8] Sboui, S. (2018). Female factor infertility. MOJ Biology and Medicine, 3(1), 9-10. https://doi.org/10.15406/mojbm.2018.03.00066

[9] Hope, Y.Y., Roxanne, V., \& Gary, N. 2017. Frishman. "Female Infertility." Clinical Reproductive Medicine and Surgery. Springer, Cham. 227-241.

[10]Zarins, D.M. 2018. Methods and systems for the manipulation of ovarian tissues. U.S. Patent Application No 15/562,861.

[11] Apirakviriya, C., Rungruxsirivorn, T., Phupong, V., Wisawasukmongchol, W. 2016. Diagnostic accuracy of 3D-transvaginal ultrasound in detecting uterine cavity abnormalities in infertile patients as compared with hysteroscopy. European Journal of Obstetrics \& Gynecology and Reproductive Biology, 200:

24-28.

https://doi.org/10.1016/j.ejogrb.2016.01.023

[12] Hussain, N., \& Das, R. 2018. Transvaginal Ultrasound Findings Among the Women Presenting with Infertility. Chattagram Maa-OShishu Hospital Medical College Journal, 16(2), 31-34.

[13] Anwar, S., \& Anwar, A. 2016. Infertility: a review on causes, treatment and management. Women's Health Gynecol 5 (2016).

[14] Purohit, P., Vigneswaran, K., Fibroids \& Infertility. 2016. Curr Obstet Gynecol Rep 5(2016): 81-88. https://doi.org/10.1007/s13669-016-0162-2 